\title{
VERIFICACIÓN DEL DISEÑO DE TRATAMIENTOS SUPERFICIALES MEDIAN- TE EL MÉTODO MODOT T 72 Y LA SECCIÓN 409 DEL ESTADO DE MISSOURI Y SU POSIBLE INCORPORACIÓN A LA NORMATIVA ARGENTINA
}

\author{
DESIGN VERIFICATION OF SURFACE TREATMENTS THROUGH TEST METHOD \\ MODOT T 72 AND SECTION 409 FROM THE STATE OF MISSOURI AND ITS \\ POSSIBLE INCLUSION TO ARGENTINIAN STANDARDS
}

\author{
Cecilia J., Soengas Chaparro
}

Ing. Responsable del Área Materiales Viales. LEMaC Centro de Investigaciones Viales, Universidad Tecnológica Nacional, Facultad Regional La Plata, Buenos Aires, Argentina.

E-mail: csoengas@frlp.utn.edu.ar

Gerardo, Botasso Campagno

Mg. Ing. Director del LEMaC, LEMaC Centro de Investigaciones Viales, Universidad Tecnológica Nacional, Facultad Regional La Plata, Buenos Aires, Argentina.

E-mail: gbotasso@frlp.utn.edu.ar

Oscar R. Rebollo Roser

Responsable Técnico del Área Materiales Viales, LEMaC Centro de Investigaciones Viales, Universidad Tecnológica Nacional, Facultad Regional La Plata, Buenos Aires, Argentina.

Chrsitian G., Piermaria Chas

Técnico Becario, LEMaC Centro de Investigaciones Viales, Universidad Tecnológica Nacional, Facultad Regional La Plata, Buenos Aires, Argentina.

Fecha de recepción: 28 de septiembre de 2009

Fecha de aceptación: 20 de noviembre de 2009

\section{RESUMEN}

El presente artículo tiene por objeto observar la relación entre las especificaciones citadas en el Departamento de Transporte de Materiales de Construcción del Estado de Missouri, Estados Unidos "Test Method MoDOT T 72 Sweep Test of Bituminous Surface Treatment Simple" y la Sección 409 "Seal Coat" con las especificaciones establecidas en el Pliego de la Dirección Nacional de Vialidad de la República Argentina, considerando además adecuaciones surgidas en el LEMaC, a efectos de lograr mayor representatividad en relación a la obra. Para llevar a cabo la correlación de lo citado, se diseñó un tratamiento superficial simple con el método de dosificación propuesto por el Ing. Tagle, conocido como la Regla de 9-5-3 y una capa de sello con arena de tritura- 
ción. Al tratamiento se lo somete al ensayo de barrido, y teniendo en cuenta los valores citados en la Sección 409 del MoDOT, se desea valorar la eficiencia del método de dosificación optado. Se analizaron los resultados obtenidos y se comparó si el método de dosificación con lo estipulado por la Sección 409 de acuerdo a la granulometría de la capa de sellado y las pérdidas derivadas del ensayo. En las Especificaciones Nacionales no existe una restricción numérica de dichas pérdidas sino solo la experiencia de obra y los resultados de ensayos cuando el tratamiento ya está colocado como lisura superficial, rugosidad y coeficiente de fricción, además de los parámetros de los materiales separadamente. Se ha logrado con la experimentación analizada, establecer una recomendación para su posible incorporación a los Pliegos citados.

Palabras clave: Tratamiento Superficial; Test del Barrido; Normativa.

\begin{abstract}
This paper verifies the relations among the specifications given by the Construction Materials and Transport Department of the State of Missouri, United States -Test Method MoDOT T 72 Sweep Test of Bituminous Surface Treatment Sample- and Section 409 -Seal Coat- with the established specifications in the National Road Department folder from Argentina, considering further adjustments arising in the LEMaC in order to get a higher representation in relation to the field. For that purpose, it was designed a simple surface treatment using the dosage method proposed by Tagle -known as the 9-5-3 Rule- and a seal coat with crushing sand. This surface treatment is submitted to the Sweep Test, and taking into account the values given by the Section 409 from MoDOT, the dosage method efficiency is evaluated. The results were analyzed and a comparison was made between the dosage method and the specifications given in Section 409 -according to the seal coat gradation and the losses from the test. National Specifications have no numerical restrictions of such a loss, excepting the field experience and the test results where the surface treatment is already used to produce absence of roughness on a surface and the friction coefficient, besides the material parameters. Based on the experimentation tested a recommendation is given for a possible inclusion into the specifications referred.
\end{abstract}

Keywords: surface treaments; sweep test; standards.

\title{
INTRODUCCIÓN
}

Los tratamientos superficiales están compuestos en su gran mayoría por una sucesión de riegos de emulsión bituminosa y la distribución de agregados pétreos. Dependiendo de su función es la elección granulométrica de los agregados distribuidos y por ende de la capa de sellado. Existen distintos tipos de tratamientos superficiales como los 
denominados simples, dobles y triples. También están los denominados tratamientos bituminosos que no necesariamente se formulan con la inclusión de agregado pétreo.

La mayor utilidad que se le da a los tratamientos superficiales en el ámbito vial nacional, es la mejora de las características superficiales de caminos de bajo tránsito. La estructura de los tratamientos no es el resultado de consideraciones científicas sino de las experiencias prácticas y observaciones en obra. Es por tal motivo que desde trabajos anteriores del LEMaC, Centro de Investigaciones Viales, se planteó la necesidad de verificar el método adoptado para dosificar tratamientos superficiales, en particular el tipo simple.

Para ello se tomó el ensayo propuesto por el MoDOT T 72, siguiendo los lineamientos generales basado en la Norma ASTM D 7000 "Standard Test Method of Sweep Test of Bituminous Emulsion Surface Treatment Simples" la cual especifica el corte efectivo de la emulsión, simulando un barrido en su superficie calculando luego la pérdida de agregado en gramos del tratamiento. La Sección 409 del MoDOT, estipula entre otros ítems, la cantidad en gramos de pérdida de agregados de la capa de sello, dependiendo del tipo de granulometría utilizada en el tratamiento.

A pesar de que el ensayo MoDOT T 72 no es para verificar dicha pérdida se vio conveniente tomar la metodología para valorar la dosificación.

En el diseño del tratamiento superficial, en vez de colocar el mismo, como lo propone el método MoDOT T 72 sobre un fieltro asfáltico, se utilizó una base típica granular como lo estipula el Pliego de Especificaciones Técnicas Generales de la Dirección Nacional de Vialidad en su última edición de 1998.

\section{MATERIALES}

En los tratamientos tanto el agregado grueso como el fino utilizado en el presente trabajo proviene de canteras cercanas a la ciudad de Olavarría, provincia de Buenos Aires. Del análisis petrográfico de ambos se advierte que el agregado grueso corresponde a un granito feldespático y el agregado fino es una arenisca feldespática (tabla 1). En la figura 1 y 2 se pueden apreciar el agregado grueso y el fino.

Tabla 1. Composición Mineralógica de los agregados pétreos.

\begin{tabular}{|l|c|c|c|c|c|}
\hline \multicolumn{1}{|c|}{ Muestra } & Cuarzo (\%) & $\begin{array}{c}\text { Feldespato } \\
\text { Potásico (\%) }\end{array}$ & $\begin{array}{c}\text { Plagioclasa } \\
(\%)\end{array}$ & Piroxeno (\%) & Otros (\%) \\
\hline $\begin{array}{l}\text { Granito } \\
\text { Feldespático }\end{array}$ & 40.2 & 47.7 & 4.1 & 5 & 3 \\
\hline $\begin{array}{l}\text { Arenisca } \\
\text { Feldespática }\end{array}$ & 35 & 47.6 & 6.2 & 5.2 & 6 \\
\hline
\end{tabular}




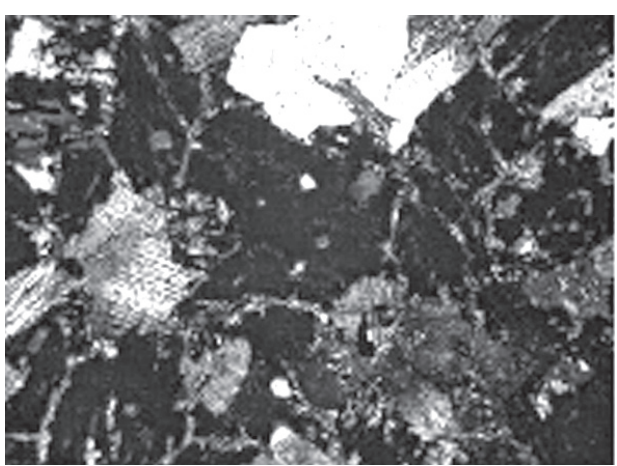

Figura 1. Agregado grueso.

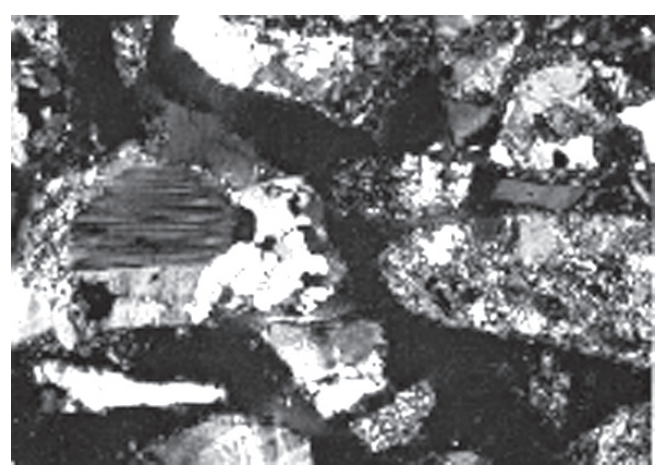

Figura 2. Agregado fino

Los resultados de los ensayos de caracterización del agregado grueso se presentan en la Tabla 2. La granulometría del agregado grueso fue realizada según lo estipulado por el Pliego de Especificaciones Técnicas Generales de la Dirección Nacional de Vialidad en su sección D.IV "Tratamientos bituminosos superficiales tipo simple”, propuesto en la sección 1003 "Aggegate For Seal Cotas” del MoDOT y la Norma IRAM 1684/74 "Agregados para tratamientos superficiales bituminosos simples y múltiples".

Tabla 2. Caracterización de los agregados pétreos gruesos

\begin{tabular}{|l|c|c|}
\hline \multicolumn{1}{|c|}{ Ensayo } & Norma & Resultado obtenido \\
\hline Índice de elongación & IRAM 1687 - parte 2 & $50 \%$ \\
\hline Índice de lajosidad & IRAM 1687 - parte 1 & $12 \%$ \\
\hline $\begin{array}{l}\text { Resistencia al Desgaste con la máquina } \\
\text { Los Ángeles }\end{array}$ & IRAM 1532 & $30,30 \%$ \\
\hline $\begin{array}{l}\text { Determinación del material fino que pasa } \\
\text { por el tamiz IRAM 75 } \mu \text { m por lavado }\end{array}$ & IRAM 1540 & $1.46 \%$ \\
\hline Análisis Mecánico de materiales granulares & VN - E - 7 - 65 & $0.47 \%$ \\
\hline Determinación del polvo adherido & VN - E $68-75$ & $0.75 \mathrm{ml} / 100 \mathrm{ml}$ \\
\hline $\begin{array}{l}\text { Determinación de la densidad relativa real, } \\
\text { densidad relativa aparente y de la absor- } \\
\text { ción de agua }\end{array}$ & IRAM 1533 & $\begin{array}{c}\text { Densidad relativa aparente }= \\
2.678 \mathrm{gr} / \mathrm{cm}^{3}\end{array}$ \\
\hline $\begin{array}{l}\text { Determinación de la densidad a granel } \\
\text { y de los espacios vacíos }\end{array}$ & IRAM 1548 & Absorción $=0.25 \%$ \\
\hline
\end{tabular}

De todas las curvas confeccionadas, se optó por la correspondiente a la norma IRAM 1684, curva 6 de la figura 3, debido a que, por el tipo de agregado, las otras no encuadraban. 


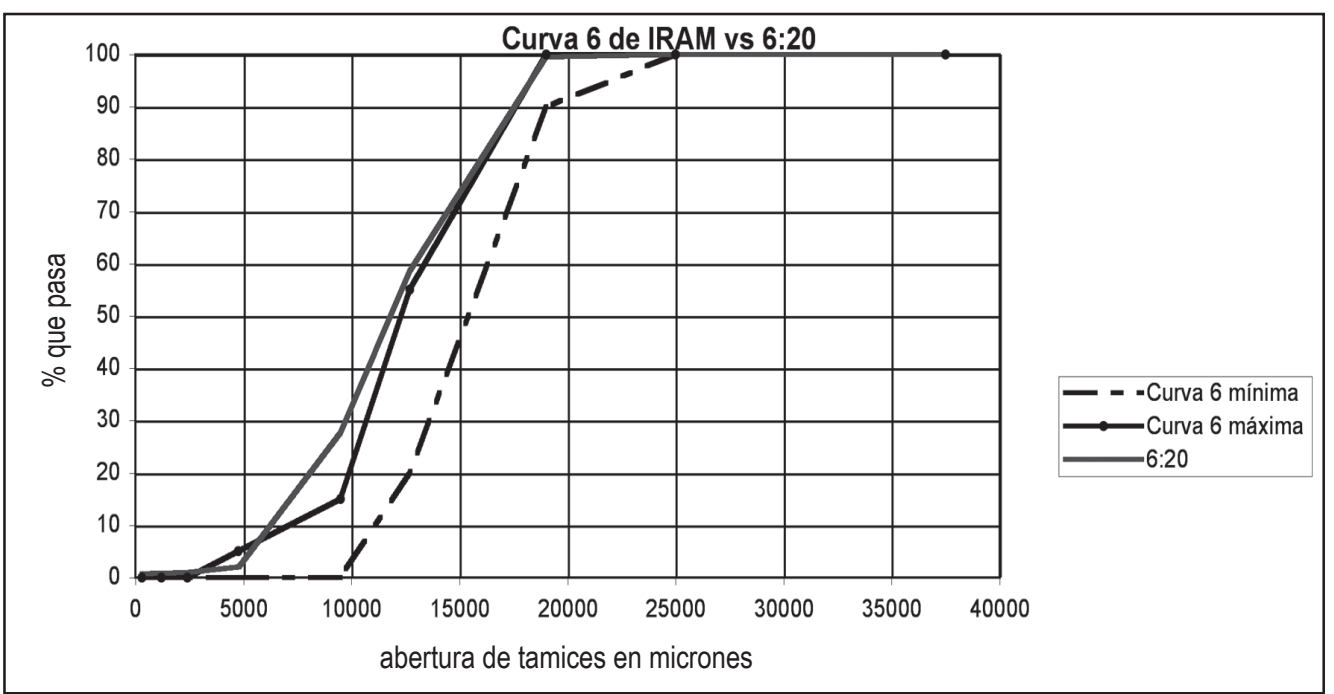

Figura 3. Curva granulométrica del agregado grueso y la curva límite 6.

El agregado fino utilizado en la capa de sellado fue estudiado de acuerdo a lo estipulado en la sección D.III "Tratamiento bituminoso superficial de sellado" del Pliego de Especificaciones Técnicas Generales de la Dirección Nacional de Vialidad y la sección 409 del MoDOT. Los resultados de la caracterización física del mismo se dan en la Tabla 3.

Tabla 3. Caracterización de los agregados pétreos finos

\begin{tabular}{|l|l|c|}
\hline \multicolumn{1}{|c|}{ Ensayo } & Norma & Resultado Obtenido \\
\hline $\begin{array}{l}\text { Determinación del material fino que pasa por el tamiz } \\
\text { IRAM 75 } \mu \mathrm{m} \text { por lavado }\end{array}$ & IRAM 1540 & $9.06 \%$ \\
\hline Análisis Mecánico de materiales granulares. & VN $-\mathrm{E}-7-65$ & $0.53 \%$ \\
\hline $\begin{array}{l}\text { Determinación de la densidad a granel y de los es- } \\
\text { pacios vacíos. }\end{array}$ & IRAM 1548 & $1.543 \mathrm{gr} / \mathrm{cm} 3$ \\
\hline
\end{tabular}

De todas las curvas granulométricas confeccionadas, se eligió la correspondiente a la curva C (figura 4) del Pliego de de Especificaciones Técnicas Generales de la Dirección Nacional de Vialidad. 


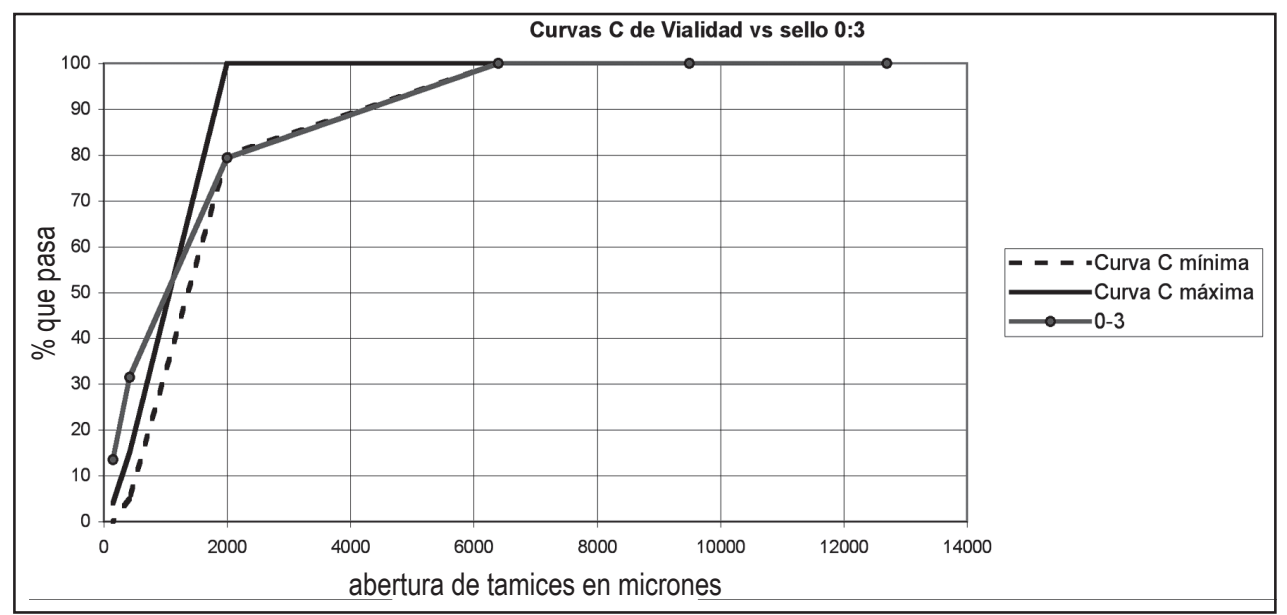

Figura 4. Curva granulométrica del agregado fino y la curva límite $C$

En referencia a la emulsión utilizada en el presente trabajo, se usó una de rotura rápida catiónica modificada debido a la eficiencia conocida de los ligantes modificados comparándolos con los convencionales. El tipo de rotura elegido se debe a que en el proceso constructivo, dependiendo del tipo de clima y de la base que se vaya a proteger, es necesario que despues del riego de emulsión se distribuyan los agregados pétreos antes del corte de la emulsión.

En el caso que la relación pasa tamiz № 200 (Abertura IRAM $75 \mu \mathrm{m}$ ) vía seca y húmeda no cumpla con lo estipulado en el Pliego de de Especificaciones Técnicas Generales de la Dirección Nacional de Vialidad o existan condiciones climatológicas muy adversas para el corte de la emulsión (es decir muy ventoso y/o seco) la recomendación de la mayoría de la bibliografía consultada y los Pliegos de Especificaciones, es la utilización de emulsión de corte medio. Según lo expuesto y de acuerdo con la caracterización del fabricante de la emulsión, se adjunta en la Tabla 4 los resultados de los ensayos.

Tabla 4. Caracterización de la emulsión asfáltica catiónica modificada

\begin{tabular}{|l|c|c|c|c|}
\hline \multicolumn{1}{|c|}{ Ensayos de la emulsión } & Unidad & Resultados & Rango & Norma \\
\hline Viscosidad Saybolt - Furol a $25^{\circ} \mathrm{C}$ & SSF & 18 & $>20$ & IRAM 6721 \\
\hline Betún Asfáltico residual & \% en peso & 65 & $\geq 63$ & IRAM 6719 \\
\hline Sedimentación en 7 días & $\%$ en peso & 1.2 & $<5$ & IRAM 6716 \\
\hline Fluidificantes por destilación & $\%$ en peso & 0.0 & $<5$ & IRAM 6219 \\
\hline pH de la emulsión & --- & 2.2 & $<7$ & NLT 195/92 \\
\hline Residuo sobre tamiz IRAM $850 \mu \mathrm{m}\left(\mathrm{N}^{0} \mathrm{20}\right)$ & $\%$ en paso & 0.0 & 0.1 & IRAM 6717 \\
\hline
\end{tabular}


Con respecto al estabilizado granular utilizado, fue estudiado según lo estipulado por la Sección C.II "Bases o sub - bases de agregados pétreos y suelo" del Pliego de de Especificaciones Técnicas Generales de la Dirección Nacional de Vialidad. Valor soporte superior al $80 \%$ y curva tipo B.

Para la dosificación de un tratamiento superficial, existen métodos teóricos, prácticos y empíricos, dentro de este último se desprenden distintas propuestas. Del análisis bibliográfico, se optó por el más difundido y utilizado en el país que es la denominada Regla 9-5-3 propuesto por el Ing. E. F. Tagle. Este método figura dentro de los empíricos y será el que describiremos.

\subsection{CONSIDERACIÓN TEÓRICA DE LA DOSIFICACIÓN}

La regla de Tagle determina la cantidad de ligante en los tratamientos simples, dobles y triples, en función de las cantidades y características granulométricas de los agregados pétreos. Las premisas que se deben tener en cuenta son: no se contempla la dosificación de sellado y retratamientos; la base es suficientemente estable y sin exceso de material bituminoso imprimado; los agregados pétreos son de una cubicidad aceptable; la cantidad de agregado pétreo en los tratamientos tipo simple no excede en más de un $20 \%$ de la necesaria para cubrir la superficie a tratar; sobre los tratamientos tipo doble y triple se aplicará un riego de sellado a razón de $0.7 \mathrm{l} / \mathrm{m}^{2}$ de emulsión bituminosa. El método se basa en los siguientes lineamientos:

a) Para la cantidad de agregado pétreo se tiene en cuenta el método práctico pero en vez de utilizar $1 \mathrm{~m} 2$ se usa $1 / 4$ de $\mathrm{m} 2$, el mismo puede ser un tablero o tela.

b) La cantidad de material bituminoso, expresada en volumen de cemento asfáltico, reducido a $15,5^{\circ} \mathrm{C}$ de temperatura, está relacionada con el volumen del agregado pétreo suelto, por los porcentajes expresados en la Tabla 5.

c) Cuando en vez de cemento asfáltico se emplean emulsiones bituminosas, será necesario aumentar las cantidades con el fin de igualar el contenido de cemento asfáltico calculado.

Tabla 5. Descripción de la Regla de Tagle

\begin{tabular}{|l|c|l|c|}
\hline \multicolumn{1}{|c|}{ Tratamiento Tipo } & $\begin{array}{c}\text { Aplicación } \\
\text { Bituminosa }\end{array}$ & \multicolumn{1}{|c|}{ Relaciones } & Porcentaje \\
\hline Simple, Doble y Triple & Total & Betún -Piedra (en volumen de agregado suelto) & 9 \\
\hline Doble y Triple & $1^{0}$ Riego & $\begin{array}{l}\text { Betún - Tamaño Máximo Efectivo }{ }^{1} \text { (del agregado } \\
\text { grueso) }\end{array}$ & 5 \\
\hline Triple & $2^{\circ}$ Riego & $\begin{array}{l}\text { Betún - Agregado Grueso (en volumen de agrega- } \\
\text { do grueso) }\end{array}$ & 3 \\
\hline
\end{tabular}

(1) Se entiende por tamaño máximo efectivo de un agregado, el número de milímetros correspondientes al valor del $80 \%$ en su curva granulométrica de porcentajes en peso que pasan. 


\section{ESPECIFICACIONES TÉCNICAS NACIONALES Y SUS CARACTERÍSTICAS}

\subsection{ANÁLISIS DEL PLIEGO DE ESPECIFICACIONES TÉCNICAS GENERALES DE LA DIRECCIÓN NACIONAL DE VIALIDAD}

La Sección D.I "Disposiciones generales para la ejecución de imprimación, tratamientos superficiales, bases, carpetas y bacheos bituminosos" del Pliego de Especificaciones Técnicas Generales de la Dirección Nacional de Vialidad, detalla las características constructivas; características físicas de cada uno de los materiales que intervienen; cómo deberán ser tomadas las muestras de dichos materiales para que sean representativos para su evaluación y cuáles serán las condiciones para la recepción; equipos utilizados; fallas del tratamiento superficial; en el apartado D.I 3 "Fórmulas para las mezclas asfálticas y tratamientos bituminosos superficiales" solo se cita que: .... dicha fórmula debería ser verificada por la supervisión con las características de calidad de los agregados y del ligante asfáltico, así como las proporciones de los mismos para los distintos tipos de riegos, incluyendo posible incorporación de aditivos, página 129 del citado Pliego. En el apartado D.I 5.9 "Composición del tratamiento superficial" cita: ... estos controles se realizarán directamente en cancha durante la ejecución de los riegos asfálticos y distribución de agregados, salvo que en el pliego particular se prevean otros ensayos... Página 143 del Pliego.

Por todo lo expuesto, conviene que la fórmula de obra sea aprobada antes y realizada fuera del área de trabajo.

En la sección D.III "Tratamientos superficiales de sellado" en el apartado D.III.2.1 "Materiales bituminosos", en la página 149 se cita: ...El riego de material bituminoso se hará con emulsión catiónica de rotura rápida o media, a razón de 0.4 a 0.9 litros por metro cuadrado de residuo asfáltico. Como valor orientativo debe utilizase el valor de 0.10 para la relación betún - agregado en volumen, a partir de la cual el contratista en una sección de prueba de longitud aproximada de $200 \mathrm{~m}$, verificará tras un librado al tránsito no menor de 15 días.

En las páginas 149 y 150 del apartado D.III 2.2 "Agregado" cita: ...El agregado pétreo se distribuirá a razón de 3 a 7 litros por metro cuadrado, y su granulometría estará comprendida dentro de los límites que se expresan en la Tabla 6. 
Tabla 6. Granulometría propuesta para la utilización de la capa de sellado en tratamientos bituminosos superficiales

\begin{tabular}{|c|c|c|c|c|c|c|}
\hline \multirow[b]{2}{*}{ Tipo } & \multicolumn{6}{|c|}{ \% Pasa Tamiz } \\
\hline & $\begin{array}{c}1 / 2 " \\
12.7 \mathrm{~mm}\end{array}$ & $\begin{array}{c}3 / 8 " \\
9.5 \mathrm{~mm}\end{array}$ & $\begin{array}{c}1 / 4^{\prime \prime} \\
6.4 \mathrm{~mm}\end{array}$ & $\begin{array}{c}\mathrm{N}^{0} 10 \\
2.0 \mathrm{~mm}\end{array}$ & $\begin{array}{c}\mathrm{N}^{0} 40 \\
0.42 \mathrm{~mm}\end{array}$ & $\begin{array}{c}\mathrm{N}^{0} 100 \\
0.15 \mathrm{~mm}\end{array}$ \\
\hline$A$ & 100 & $95-100$ & $60-85$ & $5-20$ & $0-3$ & --- \\
\hline$B$ & --- & 100 & $90-100$ & $20-50$ & $0-10$ & $0-2$ \\
\hline C & --- & --- & 100 & $80-100$ & $5-15$ & $0-4$ \\
\hline
\end{tabular}

Fuente: Pliego de Especificaciones Técnicas Generales de la Dirección Nacional de Vialidad

Los agregados pétreos y el tipo de granulometría que se va a emplear en la obra se indicarán en la Especificación Particular. Como dato ilustrativo pueden admitirse los siguientes valores por metro cuadrado: Tipo A: 5 a 7 litros, Tipo B: 3 a 4.5 litros y Tipo C: 3 litros.

La Supervisión podrá verificar en cualquier momento las cantidades fijadas por el Contratista mediante el Ensayo manual de "cubrimiento" directamente sobre la superficie tratada...

El ensayo de "Cubrimiento" se refiere a la valoración de la adhesividad del par ligante - agregado, estipulado por la norma IRAM-1804 "Agregados. Adhesividad mediante la placa Vialit".

\subsection{ANÁLISIS DEL PROCEDIMIENTOS MODOT}

El Departamento de Materiales de la Construcción y del Transporte (MoDOT) del Estado de Missouri de los Estados Unidos, basados en la norma ASTM D 7000 - 04 "Método de Ensayo Normalizado para el Test de Barrido de Tratamientos Superficiales de Emulsiones Bituminosas" y en la ASTM WK 139 "Nuevo Método de Ensayo para el Ensayo de Barrido de Muestras de Tratamientos superficiales Bituminosos" propone los Ensayos MoDOT T 72, en correspondencia con la ASTM D 7000, en la sección 409 Capa de Sellado y en la sección 1003 los husos granulométricos de los tratamientos.

El ensayo MoDOT T 72 mide las características del rendimiento del material bituminoso mediante la simulación de un tratamiento superficial durante la operación del barrido (punto 1.0 Alcance).

Para realizar el barrido, se utiliza la máquina Hobart modelo A 120 para la abrasión de un cepillo con determinadas características de dimensiones, pesos y materiales (las cerdas del cepillo deberán ser de nylon).

La confección de la pastilla de tratamiento se realiza con material bituminoso, agregados de determinada granulometría y una base de fieltro asfáltico de características 
especificadas. El acondicionamiento de la muestra y el procedimiento de ensayo están especificados en los apartados 5.0 y 6.0 .

El cálculo de las pérdidas obtenidas antes y luego del barrido tiene en cuenta la superficie barrida, la diferencia de pesadas antes y luego de la abrasión.

La sección 409, en sus apartados 409.1 y 409.2, describe las características que deben cumplir los materiales componentes de un tratamiento superficial. En el apartado 409.3 la fórmula de la mezcla de trabajo antes de la colocación de la capa de sellado. En particular nos centramos en el apartado 409.3.2 donde cita: ... el ensayo de barrido de tratamientos superficiales bituminosos deberán estar de acuerdo con los criterios expuestos en la Tabla 7 que fue utilizada para evaluar las pérdidas que sufren las pastillas de tratamiento superficial confeccionadas.

Tabla 7. Valores de pérdida del ensayo del barrido, dependiendo de la granulometría utilizada en el tratamiento superficial.

\begin{tabular}{|l|l|c|c|c|}
\hline \multicolumn{3}{|c|}{ Requerimientos del ensayo de barrido } \\
\hline \multirow{2}{*}{$\begin{array}{l}\text { Ensayo de barrido, acondicionamiento } \\
\text { durante una hora } \mathrm{a} 35^{\circ} \mathrm{C} .\end{array}$} & Método de ensayo & \multicolumn{3}{|c|}{ Granulometría } \\
\cline { 2 - 6 } & MoDOT Método de ensayo TM 72 & $\mathrm{A}$ & $\mathrm{B}$ & $\mathrm{C}$ \\
\cline { 3 - 6 } & & 15 & 40 & $\mathrm{n} / \mathrm{a}$ \\
\hline
\end{tabular}

Fuente: Sección 409, Capa de sellado, MoDOT

\section{PROPUESTA DE TRABAJO DE LABORATORIO}

La secuencia propuesta del tratamiento superficial y sus materiales, se puede sintetizar en la figura 5.

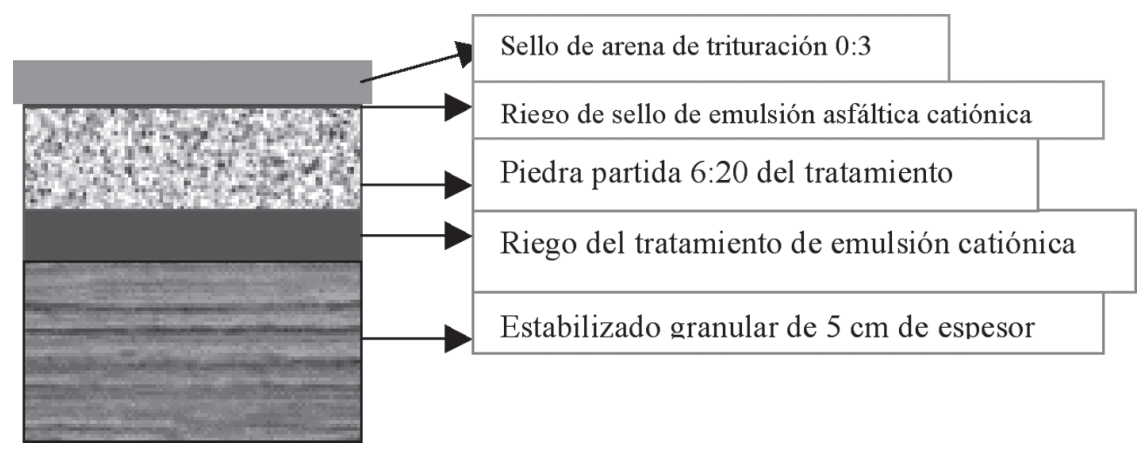

Figura 5. Esquema de trabajo de laboratorio

Para la confección de las muestras se utilizó el molde del ensayo de Weel Tracking Test, el cual tiene $5 \mathrm{~cm}$ de alto y $30 \mathrm{~cm}$ de lado. Se eligió esto en vez de realizar las 
pastillas circulares propuestas por el MoDOT debido a que se pretendió colocar el estabilizado granular de forma similar a la obra.

Las pastillas confeccionadas se regaron con distintos contenidos de emulsión asfáltica. En primer lugar se colocó la cantidad de riego que se obtuvo de la dosificación adoptada, en adelante se llamará contenido de emulsión óptimo. En el Gráfico $N^{\circ} 4$ es el $100 \%$ dicho contenido óptimo. A partir de allí se colocó un $15 \%$ en defecto y un $15 \%$ en exceso para verificar lo que sucede generalmente en obra que es el caso de exceso de riego o defecto de riego, transformándose luego en problemas constructivos como poco recubrimiento de los agregados o exudación de ligante.

Colocado el estabilizado granular con el contenido de humedad óptimo en el molde mencionado, se compacta con un martillo neumático de base cuadrada de $15 \mathrm{~cm}$ de lado. Luego de 24 horas en ambiente de laboratorio y tratando de simular las condiciones de obra, se pesa el molde más el estabilizado granular. Se procede con la aplicación del primer riego de emulsión el cual es a través de un dispersor presurizado fingiendo el regado de la barra distribuidora convencional de riego de obra. Por diferencia de pesos, se coloca la cantidad de emulsión estipulada por la dosificación. De inmediato se esparce el agregado grueso y se apisona con el compactador propuesto por el ensayo MoDOT T 72. Después se aplica el segundo riego de emulsión teniendo en cuenta lo especificado en la Sección D.III "Tratamientos Superficiales de Sellado" del Pliego de Especificaciones Técnicas Generales de la Dirección Nacional de Vialidad y a la curva granulométrica elegida para tal agregado fino. Seguido del riego se esparce el agregado de sellado, sin compactar.

La distribución de ambos agregados se realiza antes de la rotura de la emulsión. La muestra así confeccionada, se deja en ambiente de laboratorio durante 24 horas para luego someterla al ensayo de barrido. Para realizar el barrido se utiliza una máquina Hobart N 50 (usada para el ensayo de abrasión de lechadas) empleando el tiempo fijado por el MoDOT T 72.

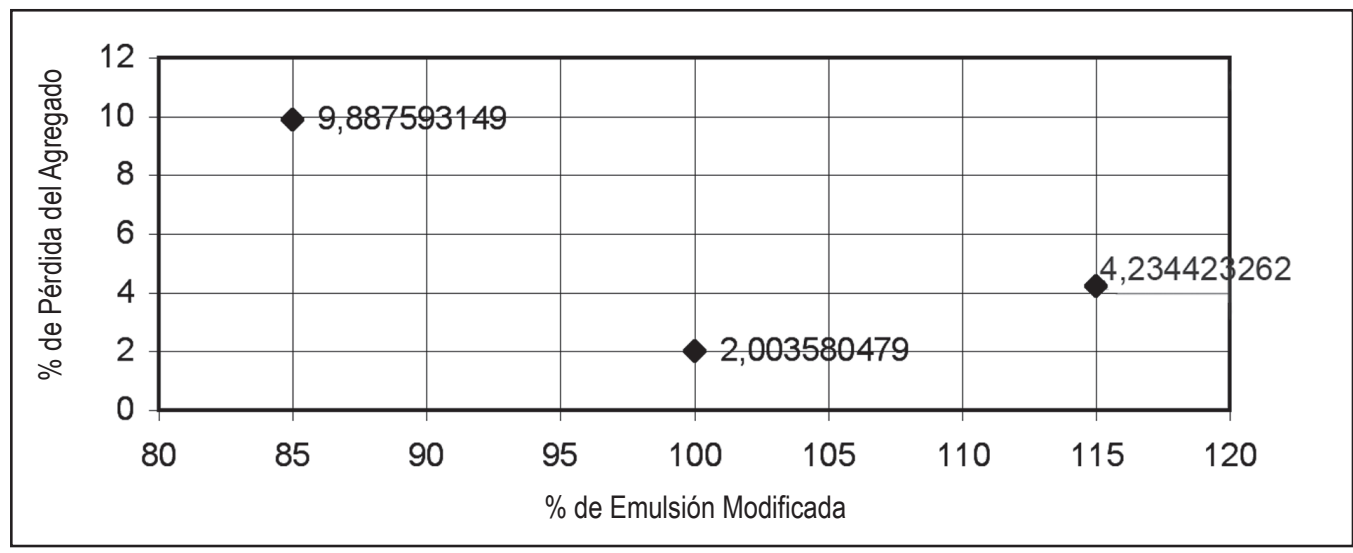

Figura 6. Valores promedios de las pérdidas del ensayo de barrido 
En la figura 6, se aprecian los valores promedios de tres determinaciones elaboradas teniendo en cuenta la máquina utilizada y el área afectada por el barrido del cepillo, los pesos iniciales y finales de cada probeta y la expresión propuesta por el MoDOT T 72 pero afectándolo con el coeficiente de barrido que corresponde al área total y la barrida.

De acuerdo con la experiencia y la información expuesta, se puede deducir que las pérdidas obtenidas con el porcentaje óptimo de emulsión, son acordes a lo estipulado por la Sección 409 del MoDOT.

\section{CONCLUSIONES}

El método de dosificación elegido para la realización de tratamientos superficiales simples es apto para el diseño de éstos, debido a las experiencias de laboratorio y a las de obras consultadas.

Se tiene buena correlación con las pérdidas obtenidas y lo especificado en las exigencias de la sección 409 del MoDOT luego del ensayo de barrido.

Se recomienda la inclusión de la prueba del barrido para la verificación de la formulación de los tratamientos superficiales como medida para la aprobación de su aplicación en obra, en el Pliego de DNV.

El exceso de riego de emulsión no implica una mayor pérdida. Para la verificación de esto se está trabajando en la realización de la prueba del Wheel Tracking Test con tratamientos superficiales con el fin de comprobar si existe exudación debido al ahuellamiento.

\section{REFERENCIAS BIBLIOGRÁFICAS}

[1] Dirección Nacional de Vialidad. Pliego de Especificaciones Técnicas Generales de la República Argentina. (1998).

[2] Laboratorio Central Des Ponts et Chaussées. Los Tratamientos Superficiales y las Rutas Económicas del Laboratorio Central Des Ponts et Chaussées, Ministerio del Equipamiento, Vivienda, Transporte y del Mar de la República Francesa. (2002).

[3] Sang Lee, Ju; Kim, Richard (2007). Entendimiento de los efectos de las proporciones de aplicación de agregado y emulsión en ele desempeño de los tratamientos superficiales asfálticos.

[4] The Asphalt Institute. Construcción de Caminos de Asfalto. Manual No 2. Tipos de Tratamientos Superficiales por "The Asphalt Institute" de Estados Unidos de Norte América. Traducido por la Dirección Nacional de Vialidad. Buenos Aires. 1939. 